\title{
Como Funciona o Xénon: Mecanismos de Neuro e Cardioprotecção
}

\author{
How Xenon Works: Neuro and Cardioprotection Mechanisms
}

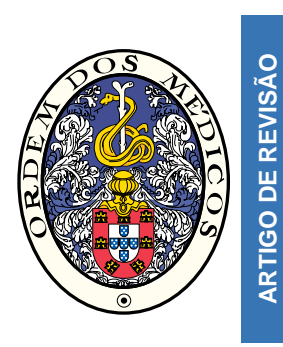

\author{
Ricardo MORAIS ${ }^{1}$, Luísa ANDRADE ${ }^{1}$, André LOURENÇO $^{2}$, Jorge TAVARES ${ }^{2}$ \\ Acta Med Port 2014 Jan-Feb;27(2):259-265
}

\section{RESUMO}

Introdução: O xénon, um gás nobre, possui qualidades anestésicas, associadas a uma notável estabilidade hemodinâmica assim como propriedades cardioprotectoras e neuroprotectoras. As suas características físico-químicas conferem-lhe uma rápida indução e emergência anestésica, estando livre de efeitos deletérios importantes nos diversos orgãos e não apresentando teratogenicidade; o que suscitou um recente recrudescimento no interesse de aprofundar o conhecimento sobre este gás nobre, afim de compreender os seus mecanismos de acção e determinar as várias indicações que possui para a prática clínica.

Material e Métodos: Revisão da literatura dos artigos considerados relevantes sobre o tema, com recurso à pesquisa de artigos indexados na Medline, com as palavras-chaves: xénon, xénon anestesia, xénon neuroproteção, xénon cardioproteção.

Resultados: A aprovação do uso do xénon em doentes ASA I-II, ocorreu em Março 2007, após a realização de dois ensaios clínicos aleatorizados multicêntricos. No entanto, o seu uso na prática clínica, tem sido limitado pelo seu preço elevado. Parece pouco provável que as vantagens que oferece em relação aos restantes anestésicos justifique o seu uso em doentes ASA I-II. No entanto, poderá ser uma preciosa ajuda para a redução das co-morbilidades e mortalidade na anestesia de doentes ASA III-IV. As suas propriedades neuro e cardio-protectoras, são também alvo de intensa investigação, com resultados promissores.

Discussão: Infelizmente, ainda não existem estudos de aleatorizados e multicêntricos que comprovem um perfil favorável do custobenefício do xénon em doentes ASA III-IV, em relação aos demais anestésicos.

Conclusão: O lugar do xénon na Anestesiologia ainda se encontra por definir.

Palavras-chave: Anestésicos Inalatórios; Fármacos Neuroprotectores; Precondicionamento Isquémico Miocárdico ; Sistema Cardiovascular Xénon.

\section{ABSTRACT}

Introduction: The Xenon, a noble gas, has anesthetics properties, associated with remarkable hemodynamic stability as well as cardioprotective, neuroprotective proprieties. Its physicochemical characteristics give him a quick induction and emergence of anesthesia, being free of deleterious effects in all organs and showing no teratogenicity. Such properties have led to a growing interest in improving the knowledge about this noble gas, in order to assess the mechanisms of neuro and cardioprotection induced and to assess the clinical indications for its use.

Material and Methods: Qualitative review of clinical trials on anesthesia with xenon. Studies were identified from MEDLINE and by hand-searching, using the following keywords: xenon, xenon anestesia, xenon neuroprotection, xenon cradioprotection.

Results: After several studies, including two randomized multicenter controlled trials, the use of xenon as an anesthetic in patients ASA I-II was approved in March 2007. However his use in clinical practice has been strongly limited by it's high price. It seems unlikely that the advantages it offers in relation to other anesthetics justify it's use in patients ASA I-II. Although, xenon may be a valuable asset in the reduction of co-morbilities and mortality in anesthesia of patients ASA III-IV, unfortunately, there are no large randomized control studies to prove it.

Discussion: Unfortunately, there are still no randomized or multicentric studies showing a favourable cost-benefit profile of xenon in ASA III-IV patients vs. other anaesthetics.

Conclusion: The usefulness of xenon in Anesthesiology requires more studies to be defined.

Keywords: Anesthetics, Inhalation; Cardiovascular System; Ischemic Preconditioning, Myocardial; Neuroprotective Agents; Protective Agents; Xenon.

\section{INTRODUÇÃO}

Os gases nobres possuem propriedades anestésicas reconhecidas desde há mais de cinquenta anos, contudo, a sua aplicação clínica é uma prática recente. ${ }^{1} \mathrm{O}$ xénon foi identificado em 1898 pelos químicos britânicos Sir William Ramsay (Prémio Nobel da Química, em 1904) e Morris Travers, ${ }^{2}$ e em 1951 foi utilizado pela primeira vez como anestésico geral em seres humanos, por Cullen e Gross. ${ }^{3}$ Mais de 6,5 milhões de litros de xénon são produzidos anualmente dos quais apenas cerca de $5 \%$ são usados na prática médica; sendo que o seu uso na anestesiologia destaca- -se como o principal responsável pelo consumo médico de xénon. ${ }^{4,5}$

Nas duas últimas décadas recrudesceu o interesse no xénon, tendo vindo a ser investigados os seus mecanismos de acção e propriedades como anestésico geral. A realização de dois ensaios clínicos aleatorizados multicêntricos foi determinante para a aprovação, em Março de 2007, do seu uso como anestésico, pela European Medicines Agency (EMEA) em doentes com classificações de estado funcional I e II da American Association of Anesthesiology (ASA). ${ }^{6}$

1. Departamento de Imagem Médica. Centro Hospitalar e Universitário de Coimbra. Coimbra. Portugal.

2. Serviço de Anestesiologia. Centro Hospitalar São João. Porto. Portugal.

Recebido: 04 de Agosto de 2013 - Aceite: 22 de Janeiro de 2014 | Copyright @ Ordem dos Médicos 2014 


\section{MATERIAL E MÉTODOS}

\section{Farmacodinâmica}

É consensualmente aceite que os anestésicos actuam por acção em receptores, destacando-se, mais frequentemente, os receptores $G_{A B A}$ inibidores da neurotransmissão excitatória, e os receptores de $\mathrm{N}$-metil-D-aspartato (NMDA), subtipo dos receptores do glutamato, ${ }^{7}$ entre outros.

O xénon não tem acção a nível dos receptores $\mathrm{GABA}_{\mathrm{A}}{ }^{7}$ Estudos recentes demonstraram que inibe de forma específica o receptor NMDA, recorrendo a métodos de cristalografia e concluíram que o xénon ocupa o mesmo local de ligação no receptor de NMDA que a glicina, sugerindo uma inibição por antagonismo, quer competitivo, quer não competitivo. ${ }^{8,9}$ Esta inibição é mais exuberante em concentrações baixas de glicina. ${ }^{8}$ Este aspecto é clinicamente relevante, uma vez que os níveis extracelulares de glicina, um co-agonista essencial à activação completa do receptor pelo glutamato, elevam-se em situações patológicas como a isquemia cerebral, in vivo. ${ }^{10}$ Banks et a ${ }^{11}$ demonstraram que o efeito neuroprotector do xénon num modelo de hipóxia in vitro, pode ser revertido com a elevação da concentração de glicina, sendo este resultado consistente com a hipótese da neuroprotecção do xénon ser mediada pela inibição do receptor NMDA no local de ligação da glicina.

Outros estudos, apontam a família de canais de potássio com dois domínios moleculares em forma de poro $\left(\mathrm{K}_{2 \mathrm{P}}\right)$, como uma importante moduladora da excitabilidade neuronal e potencial alvo do xénon. ${ }^{12,13}$ Esta família, cujo primeiro membro clonado foi o TWIK1 (two-pore domain weak inward rectifying $K^{+}$channel) ${ }^{14}$ já conta com vários outros membros, como os canais TASK 1-5 (TWIK-related acid-sensitive $K^{+}$channels) e TREK 1 e 2 (TWIK-related $K^{+}$ channels). Distingue-se dos canais de potássio clássicos, não só pela sua estrutura particular, como também pelas propriedades farmacológicas. Serão canais relativamente insensíveis à diferença de voltagem, que contribuirão essencialmente, actuando de forma tónica e basal, para o potencial de repouso da membrana, ${ }^{15}$ sendo activados em situações de stress fisiológico, nomeadamente em períodos de hipóxia, por diminuição dos níveis de ATP. A sua activação reduz a excitabilidade neuronal, funcionando assim, como um mecanismo de neuroprotecção contra insultos isquémicos. ${ }^{16} \mathrm{O}$ xénon activa estes canais, sendo então possivel este ser um dos mecanismos que the confere propriedades neuroprotectoras de pré-condicionamento contra a isquemia. ${ }^{17}$

O xénon, o protóxido de azoto e os ciclopropanos activam o canal TREK-1 mas não o canal TASK-3, contrariamente ao anestésico volátil halotano (GABAérgico) que activa os dois canais. ${ }^{18} \mathrm{~A}$ activação do canal TREK-1 reduzirá a excitabilidade neuronal por intermédio da hiperpolarização, no entanto, a relevância clínica destes canais no efeito anestésico do xénon ainda não foi provada de forma concludente. $12,13,18$

Tal como sucede com os restantes anestésicos inalatórios e intravenosos, os dados até agora recolhidos sobre os mecanismos de acção do xénon apoiam a hipótese de que não haja um alvo neuronal específico que explique todo o espectro das suas acções. Assim, é provável que o efeito anestésico e anti-nociceptivo do xénon se deva a uma multiplicidade de mecanismos complexos, dependentes de vários receptores e da interacção entre vias subcelulares.

\section{RESULTADOS}

\section{Uso clínico como anestésico}

Foram conduzidos na União Europeia dois ensaios clínicos multicêntricos controlados e aleatorizados, com o objectivo de avaliar a eficácia e a segurança do xénon como anestésico geral em doentes saudáveis. ${ }^{19,20}$

Um dos estudos, levado a cabo por Rossaint et al, ${ }^{19}$ incluiu 224 doentes (ASA I-III) e demonstrou que o xénon, numa fracção inspirada de $60 \pm 5 \%$, para além de ser seguro e não induzir alterações relevantes a nível cardiovascular, apresentava um tempo de emergência da anestesia inferior comparativamente com a combinação entre isoflurano e protóxido de azoto. O outro, da autoria de Wappler et al, ${ }^{20}$ incluiu 252 doentes (ASA I-II) e teve como objectivo avaliar a função ventricular esquerda por ecocardiografia transesofágica durante a anestesia com xénon comparativamente com a combinação isoflurano-protóxido de azoto. Os resultados deste trabalho, em consonância com os do mencionado anteriormente, revelaram que o xénon não deprime a contractilidade miocárdica, nem altera significativamente a hemodinâmica, exceptuando um efeito cronotrópico negativo, ao passo que a combinação isoflurano-protóxido de azoto exerce um efeito inotrópico negativo (com uma queda de cerca de $15,8 \%$ nos índices de contractilidade), e alterações da hemodinâmica global, com queda significativa da pressão arterial média. ${ }^{20}$ Assim, embora sejam evidentemente necessários mais ensaios clínicos aleatorizados multicêntricos, preferencialmente alargados à generalidade da população de doentes cirúrgicos, a segurança e eficácia do xénon como anestésico geral, com algumas vantagens hemodinâmicas e menores tempos de emergência da anestesia e recobro pós-anestésico foi demonstrada em doentes ASA I-II. ${ }^{19,20}$

Quanto à aplicação em unidades de cuidados intensivos, o xénon foi ainda pouco estudado. Num pequeno ensaio clínico randomizado duplamente cego que envolveu 21 doentes, o xénon manteve a constância da pressão arterial mais eficazmente que o propofol, com efeitos cardiovasculares mínimos. Bedi et al, ${ }^{21} \mathrm{com}$ base nestes resultados, levantaram a hipótese que o xénon possa ter uma aplicação clínica em casos de sépsis e choque onde outros sedativos possam provocar maior depressão miocárdica. ${ }^{21}$ Devido à sua rápida emergência da sedação, possibilita ainda uma rápida avaliação do quadro neurológico dos doentes e uma redução do tempo de internamento em $\mathrm{UCl}^{1,21}$

\section{Sistema Cardiovascular}

Como mencionado anteriormente, vários trabalhos experimentais e clínicos, demonstraram uma estabilidade cardiovascular superior e notável do xénon em comparação 
com os demais anestésicos inalatórios. ${ }^{19,20}$ Os mecanismos subjacentes a esta estabilidade cardiovascular são actualmente alvo de intensa investigação. Contrariamente aos restantes anestésicos inalatórios, o xénon exerce poucos efeitos nos principais canais iónicos, incluindo os canais de sódio $\left(\mathrm{I}_{\mathrm{Na}}\right)$, cálcio $\left(\mathrm{I}_{\mathrm{Ca}, \mathrm{L}}\right)$ e potássio $\left(\mathrm{I}_{\mathrm{K}, \mathrm{r}}\right)^{22}$ em miócitos de porco estudados in vitro. ${ }^{23}$ Também em preparações de miocárdio de suínos, foi constatado que o xénon não interfere na condução auriculoventricular. ${ }^{24}$ Sendo de frisar o facto de o xénon, em contraste com os restantes anestésicos voláteis, não afectar as respostas fisiológicas a estímulos ionotrópicos ou cronotrópicos positivos (ex: isoproterenol ou cálcio). ${ }^{25}$

Os efeitos directos do xénon no fluxo coronário e função miocárdica durante a sua administração sistémica e local foram também estudados in vivo no cão, sendo relativamente modestos comparativamente com o isoflurano. ${ }^{26}$ Assim, a administração sistémica não alterou o fluxo sanguíneo coronário, e a administração coronária directa na artéria descendente anterior não reduziu a função ventricular ou o fluxo coronário. De facto, a administração coronária directa apenas teve um efeito inotrópico negativo ligeiro para fracções elevadas, de cerca de $70 \%$ de xénon, sem que fossem, no entanto, detectadas alterações hemodinâmicas ou da função do ventrículo esquerdo, sendo estas alterações bem mais pronunciadas no caso dos anestésicos voláteis de uso mais comum. ${ }^{26}$ A estabilidade hemodinâmica e a ausência de efeito depressor miocárdico parece ser extensível ao miocárdio insuficiente, tendo sido demonstrada em estudos animais com miocárdio insuficiente, cardiomiopático $^{27}$ ou com função ventricular esquerda comprometida. ${ }^{28}$ No entanto, não há dados comprovativos desta estabilidade no miocárdio insuficiente em seres humanos.

Uma vez que os anestésicos halogenados possuem propriedades cardioprotectoras na isquemia ou lesão miocárdica, fenómeno designado por precondicionamento farmacológico, ${ }^{6}$ vários estudos têm investigado o xénon no mesmo contexto. Preckel et al observaram, em modelos animais de enfarte agudo do miocárdio in vivo, uma redução da área de enfarte com a administração de xénon, quer antes do evento isquémico, ${ }^{29}$ quer durante o período de reperfusão. ${ }^{30}$ Estas características favorecem a aplicação em doentes e situações com compromisso cardiovascular, como na cirurgia cardíaca, nas cirurgias emergentes e no choque. Todavia, a ausência de estudos de larga escala em doentes ASA III e IV impossibilita a sua aplicação neste contexto. Adicionalmente, será igualmente essencial a avaliação de resultados a médio-longo prazo.

Quanto ao efeito cronotrópico negativo, a fisiopatologia não está ainda bem definida. Hanss et al postularam um efeito simpaticolítico, com desequilíbrio do sistema nervoso autónomo favorecendo o parassimpático. ${ }^{31}$ Esta hipótese é apoiada pelos resultados do estudo de Wappler et al, ${ }^{20}$ uma vez que os níveis de adrenalina e cortisol aumentaram no grupo de doentes anestesiado com protóxido de azoto e anestésico halogenado, mas não no grupo anestesiado com xénon. ${ }^{20}$ Baumert et al avançam uma hipótese alter- nativa, advogando que o mecanismo será o de uma melhor preservação da modulação da frequência cardíaca pelo sistema nervoso autónomo, por uma menor supressão do balanço simpático-vagal. ${ }^{32}$

Ainda relativamente ao sistema cardiovascular, um aspecto fisicoquímico do xénon levantou uma preocupação extremamente relevante em termos clínicos. Atendendo à sua baixa solubilidade, o xénon poderia potencialmente formar bolhas gasosas em circulação e condicionar embolias gasosas cerebrais. ${ }^{33}$ No entanto, tanto o estudo de Lockwood et $\mathrm{al}^{34}$ como o de Jungwirth et $\mathrm{al}^{35}$ não verificaram um aumento da incidência de efeitos adversos a longo prazo, nomeadamente de lesões neurológicas, decorrentes de embolias gasosas cerebrais em doentes anestesiados com xénon.

\section{Sistema Nervoso}

O xénon tem demonstrado ter efeitos de neuroprotecção antes, durante ou após a lesão neuronal ter ocorrido. ${ }^{17,36}$

Acredita-se que as suas propriedades de pré-condicionamento, não se devam tanto à sua acção ao nível dos receptores NMDA, visto que a libertação de glutamato ocorre essencialmente durante e após a lesão neuronal ocorrer; mas deverá estar relacionada com a sua acção a nível dos canais de potássio dependentes do ATP $\left(\mathrm{K}_{\text {ATP }}\right)$, activando-os quando diminui a concentração do ATP. ${ }^{37}$ Corroborando esta hipótese, estão os dados do estudo levado a cabo por Bantel et al que demonstrou que a propriedade de pré-condicionamento do xénon se encontrava abolida pelo bloqueio dos canais $\mathrm{K}_{\text {ATP }}$ através do uso de tolbutamida. ${ }^{38}$

A activação dos receptores do glutamato está envolvida em numerosos processos patológicos. A entrada de cálcio, mediada pelos receptores NMDA, despoleta cascatas bioquímicas, que culminam na morte celular. Esta neurotoxicidade subsequente a uma activação excessiva dos receptores NMDA, foi apelidada de 'excitotoxicidade' por Olney ${ }^{39}$ e acredita-se estar na génese dos mecanismos de lesão neuronal, observados em várias formas de agressão neurológica. ${ }^{40-42}$ Atendendo ao seu efeito inibitório sobre estes receptores, tem sido investigado a capacidade neuroprotectora do xénon. In vitro, em cultura celular neuroglial de rato sob influência do xénon, foi possível demonstrar uma redução da lesão neuronal induzida pelo glutamato, NMDA, pela privação de oxigénio e de glicose mas também pelo trauma mecânico focal. ${ }^{43,44}$

In vivo, o xénon atenuou as lesões neuronais provocadas pela administração de NMDA em modelos animais ${ }^{43}$ e a administração de xénon (a 70\%) durante a oclusão transitória da artéria cerebral média, reduziu de modo significativo a extensão do enfarte em comparação com a administração de protóxido de azoto. ${ }^{45}$ Adicionalmente, a administração de xénon durante a ressuscitação cardiorrespiratória num modelo experimental de paragem cardíaca induzida no suíno, condiciona não só uma menor área de necrose neuronal e atenuação da inflamação perivascular mas também menor disfunção neurocognitiva após ressuscitação 
cardiopulmonar. ${ }^{46}$

A administração de xénon também parece ser benéfica mesmo quando a isquemia já ocorreu, porque parte da lesão pós-enfarte decorre nas zonas de penumbra, zonas viáveis mas em risco de necrose no período que se segue à isquemia aguda. ${ }^{47} \mathrm{O}$ xénon melhorará a viabilidade destas zonas, conforme já demonstrado em estudos aleatorizados de modelos animais. ${ }^{48}$ No entanto a sua eficácia ainda não se encontra suficientemente comprovada, nem foi estabelecida a janela terapêutica temporal, embora se encontra estimada a cerca de 8-12 horas após a isquemia.

Atendendo às propriedades neuroprotectoras demonstradas, tem havido interesse no estudo do xénon em episódios de hipóxia neonatal. Demonstrou-se, em modelos de asfixia neonatal em ratos, que a região de isquemia foi reduzida por pré-condicionamento com xénon a $75 \%$, assim como as disfunções neurológicas a longo termo. ${ }^{49} \mathrm{O}$ seu uso em associação com a hipotermia, após um episódio de hipoxia neonatal, demonstrou ter efeitos sinérgicos na atenuação da lesão neuronal, ${ }^{50}$ assim como o seu uso em associação com o isoflurano, um agonista $\mathrm{GABA}_{\mathrm{A}}$, que também possui propriedades neuroprotectoras. ${ }^{51}$

Como muitos outros anestésicos gerais, o xénon pode ter acção neuroprotectora por diminuição do metabolismo cerebral. ${ }^{52}$ Contudo, o xénon parece ter uma vantagem relativamente aos restantes anestésicos.
Tem uma concentração alveolar mínima (CAM) necessária para a neuroprotecção inferior à CAM anestésica, estimada em $63 \%$, na população de meia idade,$^{53}$ podendo ser, portanto, utilizado independentemente da acção anestésica.

A maioria dos antagonistas NMDA ficou aquém do esperado nos ensaios clínicos em que foi testado o seu efeito neuroprotector, porque não atravessam a barreira hemato-encefálica de modo eficaz, ${ }^{54}$ mas também por apresentarem alterações do comportamento como efeito lateral. Estas alterações de comportamento associadas, por exemplo, ao protóxido de azoto e à cetamina, devem-se a uma lesão dos neurónios piramidais no córtex límbico ${ }^{54}$ e a sobre-expressão de c-Fos é um marcador desta lesão. Ma et al verificaram que o xénon não exibe esse tipo de neurotoxicidade e não aumenta a expressão de c-Fos. ${ }^{54}$

$\mathrm{Na}$ última década, vários estudos sobre o efeito neuroprotector do xénon, nomeadamente em contexto de isquemia, têm sido publicados (Tabela 1).

O xénon altera a hemodinâmica da circulação craniana. Aumenta o fluxo sanguíneo cerebral mas preserva o mecanismo de auto-regulação. ${ }^{60} \mathrm{O}$ aumento do fluxo sanguíneo cerebral pode ser preocupante em situações de hipertensão intracraniana, no entanto, considerando as evidências actuais, baseadas em estudos levados a cabo em modelos animais, não se verifica um aumento significativo da pressão intracraniana na anestesia com xénon. ${ }^{61}$ Efectivamente,

Tabela 1 - Estudos sobre o efeito neuroprotector do xénon

\begin{tabular}{|c|c|c|c|}
\hline Modelo & Intervenção & Resultados & Estudo \\
\hline $\begin{array}{l}\text { Isquemia focal em ratos } \\
\text { recém-nascidos }\end{array}$ & $\begin{array}{l}\text { Xénon }(20 \%)+\text { hipotermia } \\
\text { assíncrona }\left(35^{\circ} \mathrm{C}\right)\end{array}$ & Redução do volume do enfarte & $\begin{array}{l}\text { Martin } \\
\text { et al }\left.\right|^{55}\end{array}$ \\
\hline $\begin{array}{c}\text { Lesão hipóxia induzida em } \\
\text { neurónios corticais de embriões } \\
\text { de ratos }\end{array}$ & Xénon $(100 \%)$ & $\begin{array}{l}\text { Protecção completa contra lesão } \\
\text { celular e prevenção da libertação } \\
\text { de glutamato devido à hipoxia }\end{array}$ & $\begin{array}{l}\text { Petzelt } \\
\text { et al }\left.\right|^{56}\end{array}$ \\
\hline Lesão hipóxia em células PC-12 & Xénon $(100 \%)$ & $\begin{array}{l}\text { Protecção completa contra lesão } \\
\text { celular e prevenção da libertação } \\
\text { de dopamina devido à hipoxia }\end{array}$ & $\begin{array}{l}\text { Petzelt } \\
\text { et al }\left.\right|^{57}\end{array}$ \\
\hline Isquemia focal (em ratos) & $\begin{array}{l}\text { Xénon }(70 \% \text { ou } 35 \%) \text { antes a } \\
\text { isquemia e durante a cirurgia. }\end{array}$ & $\begin{array}{l}\text { Melhoria tanto funcional como } \\
\text { histológica do grupo que usou xénon }\end{array}$ & $\begin{array}{l}\text { Homi } \\
\text { et } \mathrm{al}^{45}\end{array}$ \\
\hline Isquemia focal neonatal em ratos & $\begin{array}{c}\text { Xénon }(20-70 \%) \\
+ \text { hipotermia }\left(30-37^{\circ} \mathrm{C}\right)\end{array}$ & $\begin{array}{l}\text { Efeito sinergético em concentrações } \\
\text { baixas e redução modestas da } \\
\text { temperatura }\end{array}$ & $\begin{array}{l}\text { Ma } \\
\text { et al }\left.\right|^{58}\end{array}$ \\
\hline Isquemia global em suínos & $\begin{array}{c}\text { Xénon }(75 \%) 15 \text { minutos } \\
\text { antes da indução de paragem } \\
\text { cardíaca }\end{array}$ & $\begin{array}{l}\text { Níveis de glicerol mais baixos no grupo } \\
\text { xénon }\end{array}$ & $\begin{array}{l}\text { Schmidt et } \\
\text { al }^{59}\end{array}$ \\
\hline Lesão traumática no hipocampo & Xénon $(75 \%)$ durante 72 horas & $\begin{array}{l}\text { Redução da lesão primária e secundária. } \\
\text { Administração eficaz até } 3 \text { horas após } \\
\text { lesão. }\end{array}$ & $\begin{array}{l}\text { Coburn } \\
\text { et } \mathrm{al}^{44}\end{array}$ \\
\hline
\end{tabular}


num modelo animal com hipertensão intracraniana, a anestesia com xénon a $75 \%$ não a agravou, tendo mesmo meIhorado a capacidade de reduzi-la com a hiperventilação. ${ }^{62}$ Estes resultados sugerem que o xénon não está contra-indicado em doentes com hipertensão intracraniana.

\section{Outros sistemas}

Uma das vantagens do xénon é não apresentar, até ao momento actual, efeitos deletérios significativos.

A exposição prolongada ao xénon não provocou alterações hematológicas ou bioquímicas, conforme demonstrado nos dois ensaios clínicos randomizados multicêntricos que avaliaram o xénon em anestesia. ${ }^{19,20}$ Estes resultados também são consistentes com estudos prévios que não identificaram in vitro interferência na coagulação ${ }^{63}$ ou na função plaquetária. ${ }^{64}$ Também dados de estudos experimentais in vitro, sugerem que o xénon não perturba o sistema imunitário humano, preservando a capacidade anti-bacteriana dos neutrófilos e monócitos e a capacidade de produção de citocinas inflamatórias dos monócitos. ${ }^{65,66}$

O xénon parece não prejudicar a função hepática ${ }^{21,67,}$ e tem sido usado na cirurgia hepática sem efeitos adversos.

Também relativamente à função renal o xénon não parece induzir qualquer compromisso. ${ }^{21,67} \mathrm{O}$ mesmo se verifica para os parâmetros respiratórios durante a ventilação mecânica na anestesia com xénon, ${ }^{19,20}$ sendo a possibilidade de ocorrência do fenómeno de hipoxia por difusão improvável com o xénon, atendendo às suas propriedades físicas e difusibilidade. ${ }^{68}$

A baixa difusibilidade, em termos comparativos com o protóxido de azoto, por exemplo, explica a ausência de aumento da pressão intraluminal a nível intestinal na anestesia com xénon, tornando-o também um anestésico apropriado na cirurgia abdominal. ${ }^{69}$

O xénon não tem propriedades mutagénicas ou carcinogénicas, ${ }^{68}$ nem demonstrou ter efeito teratogénico ou alergénico. ${ }^{70}$

\section{DISCUSSÃO}

$\mathrm{Na}$ actualidade, o preço do xénon ainda é elevado, sendo cerca de dez vezes mais dispendioso comparativamente com os anestésicos usados correntemente. ${ }^{6,71}$ No entanto, a sua utilização em circuito fechado pode contribuir para uma diminuição importante do custo, uma vez que a quantidade absorvida pelos tecidos, atendendo à sua baixa solubilidade, é pequena. ${ }^{4}$ Encontram-se vários projectos

\section{REFERÊNCIAS}

1. Sanders $R D$, Ma D, Maze M. Xenon: elemental anaesthesia in clinical practice. Br Med Bull. 2005;71:115-35.

2. Harris PD, Barnes R. The uses of helium and xenon in current clinical pratical. Anaesthesia. 2008;63:284-93.

3. Cullen SC, Groos EC. The anesthetic properties of xenon in animals and human beings, with additional observations on krypton. Science. 1951;113:580-3.

4. Hanne P, Marx T, Musati S, Santo M, Suwa K, Morita S. Xenon: uptake and costs. Int Anesthesiol Clin. 2001;39:43-61.

5. Leclerc J, Nieuviarts R, Tavernier B, Vallet B, Scherpereel P. Anestésie au xenon: du mythe à la realité. Ann Fr Anesth Réanim. 2000;30:70-6.

6. Derwall M, Coburn M, Rex S, Hein M, Rossaint R, Fries M. Xenon: em curso com esse objectivo e algumas patentes já foram registadas. Este último aspecto leva a crer que o uso de xénon em cirurgias prolongadas e cuidados intensivos, recorrendo a circuitos fechados, possa ser economicamente competitivo, ${ }^{21}$ conforme demonstrado recentemente num estudo retrospectivo com o uso do xénon em cirurgia geral em circuito fechado. ${ }^{72}$ Nakata et al fizeram uma comparação entre o custo do xénon num circuito fechado com outros anestésicos, concluindo que se forem reduzidos os gastos no preenchimento inicial do sistema e flushing, o preço de anestesia com xénon é comparável à anestesia em circuito semi-fechado com protóxido azoto-isoflurano. ${ }^{73}$

Adicionalmente o xénon possui uma vantagem ecológica, visto ser um constituinte natural do meio ambiente, não contribui para o efeito de estufa e, portanto, para o aquecimento global do planeta. Sendo um gás inerte, não afecta a camada de ozono, em contraste com outros anestésicos, em especial o protóxido de azoto, que contribui em cerca de $0,1 \%$ para o efeito de estufa ${ }^{74}$ e cuja emissão foi já sujeita a restrições no tratado de Kyoto, ${ }^{69}$ salientando-se que muitos anestésicos voláteis que têm base em clorofluorcarbonos (CFC) serão interditos em 2030. ${ }^{69}$

\section{CONCLUSÃO}

O estudo do xénon tem dado um contributo fundamental para a compreensão alargada dos mecanismos de acção dos anestésicos gerais. A sua utilização clínica não está ainda difundida, sendo necessários vários passos para que se venha a concretizar. Será primordial elucidar, por intermédio de ensaios clínicos multicêntricos randomizados de larga escala em doentes ASA III e IV, se mantém o seu perfil de segurança e se tem realmente melhores resultados em termos de mortalidade e morbilidade que os seus congéneres, que justifiquem os custos acrescidos. $O$ desenvolvimento de técnicas e aparelhos que diminuam os seus custos de produção e utilização serão também cruciais para que o xénon ocupe um lugar relevante na Anestesiologia e na prática médica futuras.

\section{CONFLITOS DE INTERESSE}

Os autores declaram a inexistência de conflitos de interesse.

\section{FONTES DE FINANCIAMENTO}

Os autores declaram a inexistência de fontes de financiamento. recent developments and future perspectives. Minerva Anestesiol. 2009;75:37-45.

7. Franks NP, Dickinson R, de Sousa SL, Hall AC, Lied WR. How does xenon produce anaesthesia? Nature. 1998;396:324.

8. Dickinson R, Franks NP. Bench-to-bedside review: molecular pharmacology and clinical use of inert gases in anesthesia and neuroprotection. Crit Care. 2010;14:229.

9. Dickinson R, Peterson BK, Banks P, Similis C, Martin JC, Valenzuela $\mathrm{CA}$, et al. Competitive inhibition at the glycine site of the N-methyl-Daspartate receptor by the anesthetics xenon and isoflurane: evidence 
from molecular modeling and electrophysiology. Anesthesiology. 2007; 107:756-67.

10. Choi KT, Chung JK, Kwak CS, Kim HK. Effect of hypocapnia on extracellular glutamate and glycine concentrations during peri-ischemic period in the rabbit hippocampus. J Korean Med Sci. 1994;9:394-401.

11. Banks P, Franks NP, Dickinson R. Competitive inhibition at the glycine site of the $\mathrm{N}$-methyl-D-aspartate receptor mediates xenon neuroprotection against hypoxia- ischemia. Anesthesiology. 2010;112:614-22.

12. Patel AJ, Honoré E, Lesage F, Fink M, Romey G, Lazdunski M. Inhalational anesthetics activate two-pore-domain background $\mathrm{K}+$ channels. Nat Neurosci. 1999;2:422-6.

13. Patel AJ, Honoré E. Anesthetic-sensitive $2 \mathrm{P}$ domain $\mathrm{K}+$ channels. Anesthesiology. 2001;95:1013-21.

14. Lesage F, Guillemare E, Fink M, Duprat F, Lazdunski M, Romey G, et al. TWIK-1, a ubiquitous human weakly inward rectifying $\mathrm{Kp}$ channel with a novel structure. EMBO J. 1996;15:1004-11.

15. Patel AJ, Honoré E. Properties and modulation of mammalian $2 \mathrm{P}$ domain Kp channels. Trends Neurosci. 2001; 24:339-46.

16. Ballanyi K. Protective role of neuronal KATP channels in brain hypoxia. J Exp Biol. 2004;207:3201-12.

17. Bantel C, Maze M, Trapp S. Neuronal preconditioning by inhalational anesthetics: evidence for the role of plasmalemmal adenosine triphosphate-sensitive potassium channels. Anesthesiology. 2009;110:986-95.

18. Gruss M, Bushell TJ, Bright DP, Lieb WR, Mathie A, Franks NP. Twopore-domain $\mathrm{K}+$ channels are a novel target for the anesthetic gases xenon, nitrous oxide, and cyclopropane. Mol Pharmacol. 2004;65:443-52.

19. Rossaint R, Reyle-Hahn M, Schulte AM, Scholz J, Scherpereel P, Vallet $B$, et al. Xenon Study Group. Multicenter randomized comparison of the efficacy and safety of xenon and isoflurane in patients undergoing elective surgery. Anesthesiology. 2003;98:6-13.

20. Wappler F, Rossaint R, Baumert J, Scholz J, Tonner PH, van Aken H, et al. Xenon Multicenter Study Research Group. Multicenter randomized comparison of xenon and isoflurane on left ventricular function in patients undergoing elective surgery. Anesthesiology. 2007;106:463-71.

21. Bedi A, Murray JM, Dingley J, Stevenson MA, Fee JP. Use of xenon as a sedative for patients receiving critical care. Crit Care Med. 2003;31:2470-7

22. Hüneke $R$, Jüngling $E$, Skasa $M$, Rossaint $R$, Lückhoff $A$. Effects of the anesthetic gases xenon, halothane, and isoflurane on calcium and potassium currents in human atrial cardiomyocytes. Anesthesiology. 2001;95:999-1006.

23. Stowe DF, Rehmert GC, Kwok WM, Weigt HU, Georgieff M, Bosnjak ZJ. Xenon does not alter cardiac function or major cation currents in isolated guinea pig hearts or myocytes. Anesthesiology. 2000;92:516-22.

24. Marx T, Froeba G, Wagner D, Baeder S, Goertz A, Georgieff M. Effects on haemodynamics and catecholamine release of xenon anaesthesia compared with total i.v. anaesthesia in the pig. $\mathrm{Br} \mathrm{J}$ Anaesth. 1997;78:326-7.

25. Schroth SC, Schotten U, Alkanoglu O, Reyle-Hahn MS, Hanrath P, Rossaint R. Xenon does not impair the reaction of cardiac muscles bundles to positive ionotropic stimulation. Anesthesiology. 2002;96:422-7.

26. Preckel B, Ebel D, Müllenheim J, Fräßdorf J, Thämer V, Schlack W. The direct myocardial effects of xenon in the dog heart in vivo. Anesth Analg. 2002;94:545-51.

27. Hettrick DA, Pagel PS, Kersten JR, Tessmer JP, Bosnjak ZJ, Gerogieff $\mathrm{M}$, et al. Cardiovascular effects of xenon in isofluraneanesthetized dogs with dilated cardiomyopathy. Anesthesiology. 1998;89:1166-73.

28. Preckel B, Schlack W, Heibel T, Rutten H. Xenon produces minimal haemodynamic effects in rabbits with chronically compromised left ventricular function. Br J Anaesth. 2002;88:264-9.

29. Weber NC, Toma O, Wolter JI, Obal D, Mullenheim J, Perckel B, et al The noble gas xenon induces pharmacological preconditionin in the rat heart in vivo via induction of PKC-epsilon and p38 MAAPK. Br J Pharmacol. 2005;144:123-32.

30. Preckel B, Mullenheim J, Moloshavij A, Thamer V, Schlack W. Xenon ad ministration during early reperfusion reduces infarct size after regional ischemia in the rabbit heart in vivo. Anesth Analg. 2000;91:1327-32.

31. Hanss R, Bein B, Turowski $P$, Cavus E, Bauer M, Andretzke M, et al. The influence of xenon on regulation of the autonomic nervous system in patients at high risk of perioperative cardiac complications. $\mathrm{Br} \mathrm{J} \mathrm{An-}$ aesth. 2006;96:427-36.

32. Baumert JH, Hein M, Hecker KE, Satlow S, Schnoor J, Roassaint R. Autonomic cardiac control with xenon anaesthesia in patients at cardiovascular risk. Br J Anaesth. 2007;98:722-7.

33. Jungwirth B, Gordan ML, Blobner M, SchmehI W, Kochs EF, Mackensen GB. Xenon impairs neurocognitive and histologic outcome after cardio- pulmonary bypass combined with cerebral air embolism in rats. Anesthesiology. 2006;104:770-6

34. Lockwood GG, Franks NP, Downie NA, Taylor KM, Mazer M. Feasibility and safety of delivering xenon to patients undergoing coronary artery bypass graft surgery while on cardiopulmonary bypass: phase I study. Anesthesiology. 2006;104:458-65

35. Jungwirth B, Kellermann K, Blobner M, Schmehl W, Kochs EF, Mackensen GB. Cerebral air emboli differentially alter outcome after cardiopulmonary bypass in rats compared with normal circulation. Anesthesiology. 2007; 107:768-75

36. Ma D, Hossain M, Pettet GK, Luo Y, Lim T, Akimov S, et al. Xenon preconditioning reduces brain damage from neonatal asphyxia in rats. $J$ Cereb Blood Flow Metab. 2006;26:199-208

37. Bantel C, Maze M, Trapp S. Noble gas xenon is a novel adenosine triphosphate- sensitive potassium channel opener. Anesthesiology. 2010;112:623-30.

38. Bantel C, Maze M, Trapp S. Neuronal preconditioning by inhalational anesthetics: evidence for the role of plasmalemmal adenosine triphosphate-sensitive potassium channels. Anesthesiology. 2009;110:986-95.

39. Olney JW. Brain lesions, obesity, and other disturbances in mice treated with monosodium glutamate. Science. 1969;164:719-21.

40. Lipton SA, Rosenberg PA. Excitatory amino acids as a final common pathway for neurologic disorders. N Engl J Med. 1994; 330:613-22.

41. Sattler R, Tymianski M. Molecular mechanisms of calcium-dependent excitotoxicity. J Mol Med. 2000;78:3-13.

42. Hardingham GE, Bading $\mathrm{H}$. The yin and yang of NMDA receptor signalling. Trends Neurosci. 2003;26:81-9.

43. Wilhelm S, Ma D, Maze M, Franks NP. Effects of xenon on in vitro and in vivo models of neuronal injury. Anesthesiology. 2002; 96:1485-91.

44. Coburn M, Maze M, Franks NP. The neuroprotective effects of xenon and helium in an in vitro model of traumatic brain injury. Crit Care Med. 2008;36:588-95

45. Homi HM, Yokoo N, Ma D, Warner DS, Franks NP, Maze M, et al. The neuroprotective effect of xenon administration during transient middle cerebral artery occlusion in mice. Anesthesiology. 2003;99:876-81.

46. Fries M, Nolte K, Demir F, Kottmann K, Timper A, Coburn M, et al. Neurocognitive performance after cardiopulmonary resuscitation in pigs. Crit Care Med. 2008;36:842-7.

47. David HN, Leveille F, Chazalviel L, MacKenzie ET, Buisson A, Lemaire $M$, et al. Reduction of ischemic brain damage by nitrous oxide and xenon. J Cereb Blood Flow Metab. 2003;23:1168-73.

48. Zhuang L, Yang T, Zhao H, Fidalgo AR, Vizcarychipi MP, Sanders RD et al. The protective profile of argon, helium, and xenon in a model of neonatal asphyxia in rats. Crit Care Med. 2012;40:1724-30.

49. Luo $Y$, Ma D, leong E, Sanders RD, Yu B, Hossain M, et al. Xenon and sevoflurane protect against brain injury in a neonatal asphyxia model. Anesthesiology. 2008;109:782-9.

50. Thoresen M, Hobbs CE, Wood T, Chakkarapani E, Dingley J. Cooling combined with immediate or delayed xenon inhalation provides equivalent long-term neuroprotection after neonatal hypoxia-ischemia. J Cereb Blood Flow Metab. 2009;29:707-14

51. Ma D, Hossain M, Rajakumaraswamy N, Franks NP, Maze M. Combination of xenon and isoflurane produces a synergistic protective effect against oxygen-glucose deprivation injury in a neuronal-glial co-culture model. Anesthesiology. 2003;99:748-51.

52. Rex S, Schaefer W, Meyer PH, Rossaint R, Boy C, Setani K, et al. Positron emission tomography study of regional cerebral metabolism during general anesthesia with xenon in humans. Anesthesiology. 2006;105:936-43.

53. Nakata $\mathrm{Y}$, Goto $T$, Ishiguro $\mathrm{Y}$, Terui $\mathrm{K}$, Kawakami $H$, Santo $\mathrm{M}$, et al Minimum alveolar concentration (MAC) of xenon with sevoflurane in humans. Anesthesiology. 2001;94:611-4

54. Ma D, Wilhelm S, Maze M, Franks NP. Neuroprotective and neurotoxic properties of the 'inert' gas, xenon. Br J Anaesth. 2002;89:739-46.

55. Martin JL, Ma D, Hossain M, Xu J, Sanders RD, Franks NP, et al. Asynchronous administration of xenon and hypothermia significantly reduces brain infarction in the neonatal rat. Br J Anaesth. 2007:98:236-40.

56. Petzelt C, Blom P, Schmehl W, Muller J, Kox WJ. Prevention of neurotoxicity in hypoxic cortical neurons by the noble gas xenon. Life Sci. 2003;72:1909-18.

57. Petzelt C, Blom P, Schmehl W, Muller J, Kox WJ. Xenon prevents cellular damage in differentiated PC-12 cells exposed to hypoxia. BMC Neurosci. 2004;5:55.

58. Ma D, Hossain M, Chow A, Arshad M, Battson RM, Sanders RD, et al. Xenon and hypothermia combine to provide neuroprotection from neonatal asphyxia. Ann Neurol. 2005;58:182-93. 
59. Schmidt M, Marx T, Gloggl E, Reinelt $H$, Schirmer U. Xenon attenuates cerebral damage after ischemia in pigs. Anesthesiology. 2005;102:92936.

60. Schmidt M, Marx T, Kotzerke J, Luderwald S, Armbruster S, Topalidis P, et al. Cerebral and regional organ perfusion in pigs during xenon anaesthesia. Anaesthesia. 2001;56:1154-9.

61. Fukuda T, Nakayama H, Yanagi K, Mizutani T, Miyabe M, Ohshima N, et al. The effects of $30 \%$ and $60 \%$ xenon inhalation on pial vessel diameter and intracranial pressure in rabbits. Anesth Analg. 2001;92:1245-50.

62. Schmidt M, Marx T, Armbruster S, Reinelt H, Schirmer U. Effect of Xenon on elevated intracranial pressure as compared with nitrous oxide and total intravenous anesthesia in pigs. Acta Anaesthesiol Scand. 2005;49:494-501.

63. Horn NA, Hecker KE, Bongers B, Baumert HJ, Reyle-Hahn SM, Rossaint $\mathrm{R}$. Coagulation assessment in healthy pigs undergoing single xenon anaesthesia and combinations with isoflurane and sevoflurane. Acta Anaesthesiol Scand. 2001;45:634-8.

64. de Rossi LW, Horn NA, Baumert JH, Gutensohn K, Hutschenreuter G, Rossaint $R$. Xenon does not affect human platelet function in vitro. Anesth. Analg. 2001;93:635-40.

65. de Rossi K, Gott K, Horn N, Heker K, Hutschenreuter G, Rossaint R. Xenon preserves neutrophil and monocyte function in human whole blood. Can J Anesth. 2002;49:942-5.

66. de Rossi LW, Brueckmann M, Rex S, Bardershneider M, Buhre W, Rossaint $R$. Xenon and isoflurane differentially modulate lipopolysaccharide- induced activation of the nuclear transcription factor KB and production of tumor necrosis factor-alpha and interleukin- 6 in monocytes. Anesth Analg. 2004;98:1007-12.

67. Reinelt H, Marx T, Kotzerke J, Topalidis P, Luederwald S, Armbruster S, et al. Hepatic function during xenon anesthesia in pigs. Acta Anaesthesiol. Scand. 2002;46:713-6.

68. Calzia E, Stahl W, Handschuh T, Marx T, Froba G, Gerogieff M, et al. Continuous arterial $\mathrm{P}(\mathrm{O} 2)$ and $\mathrm{P}(\mathrm{CO} 2)$ measurements in swine during nitrous oxide and xenon elimination: prevention of diffusion hypoxia. Anesthesiology. 1999;90:829-34.

69. Marx T, Schmidt M, Schirmer U, Reinelt H. Xenon anaesthesia. J R Soc Med. 2000;93:513-7.

70. Lynch C 3rd, Baum J, Tenbrinck R. Xenon anesthesia. Anesthesiology. 2000;92:865-8.

71. Goto T, Nakata Y, Morita S. Will xenon be a stranger or a friend? The cost, benefit, and future of xenon. Anesthesia. 2003;98:1-2

72. Stoppe C, Rimek A, Rossaint R, Rex S, Stevanovic A, Schälte G, et al. Xenon consumption during general surgery: a retrospective observational study. Med Gas Res. 2013;3:12.

73. Nakata Y, Goto T, Niimi Y, Morita S. Cost analysis of xenon anesthesia: a comparison with nitrous oxide-isoflurane and nitrous oxide-sevoflurane anesthesia. J Clin Anesth. 1999;11:477-81.

74. Goto T. Is there a future for xenon anesthesia? / Le xenon a-t-il un avenir en anesthesie? Can J Anaesth. 2002;49:335-8. 


\section{Como Funciona o Xénon: Mecanismos de Neuro e Cardioprotecção Acta Med Port 2014:27:259-265}

Publicado pela Acta Médica Portuguesa, a Revista Científica da Ordem dos Médicos

Av. Almirante Gago Coutinho, 151

1749-084 Lisboa, Portugal.

Tel: +351 218428215

E-mail: submissao@actamedicaportuguesa.com

www.actamedicaportuguesa.com

ISSN:0870-399X | e-ISSN: 1646-0758

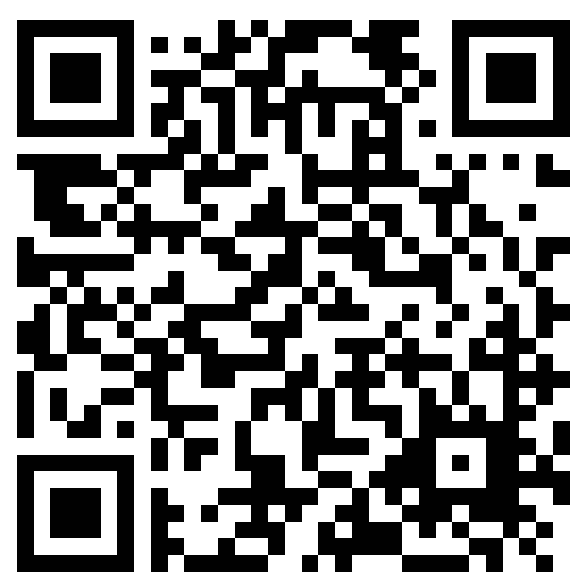

